\title{
ANÁLISIS DE LOS PROCESOS DE INCLUSIÓN SOCIAL Y EDUCATIVA: NUEVOS RETOS PARA SUPRIMIR LAS DESIGUALDADES $Y$ COMPARTIR LAS DIFERENCIAS
}

\author{
José Antonio Torres González \\ Universidad de Jaén
}

\begin{abstract}
RESUMEN: En este artículo nos planteamos analizar cómo la sociedad, a través de sus prácticas educativas, ha generado parámetros de segregación y exclusión social que después han sido reproducidos por las instituciones educativas. De esta manera, podremos entender que la sociedad no ha sido lo suficientemente integradora como en un principio, por su carácter claramente relacional, pudiera parecer, considerando a los sujetos diferentes, a la diversidad en general, como algo negativo. Esa diversidad, de los sujetos y de los colectivos humanos se ha convertido, en multitud de ocasiones, en desigualdad en la medida que las singularidades de los sujetos o de los grupos de sujetos interaccionan con las exigencias que plantea la sociedad, que ha venido utilizando los parámetros de la normalidad como síntoma de dominio, de poder y, como no, de desprecio del derecho a ser diferente.
\end{abstract}

ABSTRACT: In this article we consider to analyze as the society, across his educational practices, it has generated parameters of segregation and social exclusion that later have been reproduced by the educational institutions. Hereby, we will be able to understand that the society has not been it sufficiently of integration as in a beginning, for his clearly relational character, it could seem, considering to the different subjects, to the diversity in general, as something negative. This diversity, of the subjects and of the human groups it has turned, in multitude of occasions, in inequality in the measure that the singularities of the subjects or of the groups of subjects interdrive with the requirements that the society raises, that it has come using the parameters of the normality as symptom of domain (control), of being able and, since not, of scorn of the right to be different.

PALABRAS CLAVE: Exclusión, globalización, diversidad, desigualdad, socialización, diferencia.

KEYWORDS: Exclusion, globalization, diversity, inequality, socialization, difference. 


\section{CAMbios SOCIALES Y eXCluSión: UNA PRÁCtica EMERGENTE EN LA SOCIEDAD DE LA GLOBALIZACIÓN}

Las prácticas educativas dependen en gran medida del significado y de la valoración que tengamos de una serie de dimensiones esenciales como el individuo, la sociedad, la cultura y de cómo se relacionan entre sí. En este sentido, la educación en general, y la escuela como institución en particular, han sufrido diversos avatares debido a las condiciones cambiantes que experimenta el mundo. En la actualidad existe un conjunto significativo de investigaciones que indica con claridad que la capacidad de influencia de la escuela formal en el proceso educativo se ha debilitado de manera ostensible. Esto obedece a tres factores (Cajiao, 2001):

a) El desarrollo de los medios de comunicación, que ofrece una gama amplísima de oportunidades de información en todos los campos de la cultura, la ciencia, el entretenimiento y; sobre todo en el campo de la educación ya que "su uso permite la agilización de los procesos de enseñanza-aprendizaje, así como compartir métodos, estrategias o actividades" (Cano, 2008: 5).

b) La urbanización acelerada de la población, con la consiguiente ruptura de los patrones de organización familiar y comunitaria tradicionales; $y$,

c) La transformación de los procesos de socialización de niños y jóvenes de ambos sexos en el espacio escolar y en las extensiones de un espacio urbano mucho más accesible y atractivo.

Esta pérdida relativa de influencia no significa que la educación formal haya perdido vigencia, o que cerrar las escuelas sea la recomendación más apropiada para la época. Pero ya comienzan a verse superados los educadores por las dificultades que encuentran en relación con su función tradicional de orientadores morales, cuando sus alumnos parecen tener criterios bastante fuertes y diversos a los de ellos, junto con una clara actitud de rechazo hacia el discurso moralizador que hacía parte crucial de la educación escolar vigente hasta finales de los setenta.

En el campo del conocimiento la situación no es diferente, pues al lado de lo que dicen el maestro o el texto escolar está la enorme cantidad de información de la cual disponen los niños, aunque su nivel socioeconómico sea muy precario. Esto varía considerablemente de país a país, y, especialmente, de sector urbano a sector rural, pues la disponibilidad de información muestra una diferencia abismal en uno u otro contexto. De todos modos, la difusión de los medios de comunicación y el acceso a una gran variedad de dispositivos tecnológicos genera aprendizajes que aún no parecen llegar a la mayoría de las escuelas y de las instituciones educativas. Eso produce una asincronía muy notoria entre la enseñanza formal guiada por los currículas, que se distribuye en el ámbito de la escuela mediante pedagogías todavía muy tradicionales (enseñanza frontal, monólogo profesoral, texto guía, tareas, repetición...), y el aprendizaje informal cotidiano que se realiza en la televisión, en los juegos electrónicos interactivos y en el continuo intercambio de ideas con compañeros en espacios públicos donde no existen evaluaciones, respuestas correctas u homogeneización de intereses. Esto se incrementa en el caso de niños y jóvenes con acceso a Internet y a televisión vía satélite o de cable, pues la información se mul- 
tiplica y diversifica de una manera inimaginable para los propios adultos que interactúan con ellos.

En el terreno de la socialización, donde la escuela cumplió un papel crucial desde su origen en el siglo XVII hasta finales de la década de los setenta, hoy aparecen nuevas modalidades de relación y ocupación, especialmente en el espacio urbano por parte de niños y jóvenes, que debilitan de manera notable la influencia de la escuela formal en su función de formación de ciudadanos. En la actualidad los adolescentes gozan de mucha mayor libertad en su movilidad, lo que permite que generen actividades y organizaciones autónomas con respecto a las instituciones familiares y escolares, dando origen a tipologías de cultura juvenil que se van caracterizando de manera cada vez más definida en relación con las formas de comportamiento social, la moda, la sexualidad, el poder, la autoridad, y otra serie de comportamientos que se van distanciando cada vez más del legado de la tradición transmitida por los adultos.

Estas primeras reflexiones apuntan a mostrar la gran influencia que hoy ejercen otras instancias de la sociedad, diferentes al sistema escolar formal, en el proceso de educación integral de niños y jóvenes. Desde luego, es necesario mencionar también el papel definitivo que juega $-y$ que siempre ha jugado- el sector productivo en la educación de las personas, tanto en lo que se refiere a saberes concretos y específicos requeridos por los trabajadores, como en lo que concierne a la «cultura empresarial» que establece formas de comportamiento, disciplina, observación de normas, relaciones jerárquicas y expectativas de vida. También en este campo la crisis es muy fuerte, pues más allá de los enormes problemas de pobreza, deterioro de la calidad de vida, desempleo, trabajo informal y desinstitucionalización del trabajo profesional, el entorno laboral ejerce un indudable impacto educativo que se refleja en la organización social y en el acceso a oportunidades educativas vinculantes con el conjunto de la sociedad.

Es claro entonces, que la responsabilidad sobre la educación de la gente en una sociedad, en un país o en un municipio recae sobre el conjunto social, y esta responsabilidad debería ser asumida de manera explícita y consciente a fin de garantizar un mejor estar para niños, jóvenes y adultos, cuyo bienestar y desarrollo humano están íntimamente ligados a su posibilidad de educación permanente. Esto es lo que hace que la educación sea, por excelencia, un asunto que debe resolverse en el ámbito de lo público.

Desde luego decir que todo el mundo educa no es novedoso ni constituye un gran aporte, pues esto - de uno u otro modo- ha ocurrido siempre. Tampoco ayuda decir que todo el mundo es responsable, pues al introducir al conjunto social en una sola bolsa volvemos a quedar como al principio. Por eso el esfuerzo debe consistir en definir los campos de influencia de diversos actores sociales, intentar precisar sus papeles y sus responsabilidades, y avanzar en mecanismos prácticos que permitan que esas funciones sean asumidas de forma positiva.

Para ello conviene hacer un recorrido muy rápido por seis tópicos que me parecen fundamentales, y que, a mi juicio, siempre deberían tratarse de manera muy relacionada cuando se piensa en la educación de una comunidad, de una región o de un país, pues al fraccionarlos y mirarlos independientemente unos de otros es muy fácil 
perderse en tecnicismos o en visiones sesgadas. Los grandes núcleos de comprensión del fenómeno educativo como responsabilidad social pueden ser los siguientes:

- La responsabilidad social colectiva y el sentido de lo público en la educación.

- El papel del Estado en el desarrollo del horizonte educativo.

- La labor de los educadores y la diferencia entre la acción sindical y la acción gremial. El papel del maestro como intelectual.

- La función de la sociedad civil organizada en el desarrollo educativo.

- El influjo de los medios de comunicación e información.

- Los niños y las niñas como protagonistas de los procesos educativos y sociales.

Estos núcleos nos pueden hacer entender, como expresaba Castell (1998:42), que "una nueva sociedad surge siempre y cuando pueda observarse una transformación estructural en las relaciones de producción, en las relaciones de poder y en las relaciones de experiencia o de vida cotidiana". Cada uno de estos cambios van a tener que ver con el "equipaje humano" de las nuevas generaciones. En las relaciones de producción los cambios se aprecian en la productividad y en la competitividad. La tecnología de la información y la capacidad cultural para utilizarla son esenciales para el nuevo marco de la producción. En un sentido amplio, social, Durkheim (1975: 32) ya expresaba que "la educación no es para la sociedad sino el medio a través del cual prepara en el espíritu de los niños las condiciones esenciales de su propia existencia". Aplicada al sentido que toma hoy podemos decir que quien posee educación, en el entorno organizativo apropiado, puede adaptarse a las nuevas tareas, en cambio constante, que caracteriza a la nueva época.

En el ámbito de las nuevas formas de las decisiones políticas, lo más característico es la incertidumbre que embarga al ciudadano ante la dificultad que encuentra de delegar su voluntad en órganos con capacidad de dar respuestas. Sin embargo no por ello desaparece el poder de la nueva sociedad sino que se desempeña en un nuevo ámbito: allí donde los ciudadanos encuentran déficits estructurales para la pervivencia de su entorno, de su medio social. La política se transforma de este modo en asunto de gestión de la propia ciudadanía, tomando decisiones para lograr sus fines. Aparece así la participación como una actitud insoslayable en el nuevo ciudadano.

Pero además, las relaciones de experiencia de la vida cotidiana también cambian. Lo hacen en la misma medida que caducan las instituciones primarias tradicionales: la devaluación de la familia patriarcal simétrica donde se radicalizan los roles de género; emerge entonces la identidad de la mujer en el plano de igualdad social, convulsionando las formas básicas, fuentes directas de identidad. De estos cambios aparecen personalidades flexibles, capaces de llevar a cabo un continuo reajuste del yo -un continuo aprender- en lugar de la tradicional adaptación de manera pasiva a un sistema normativo unívoco y de roles sociales prefijado. Hoy en día las personas "producen" formas de sociabilidad en lugar de seguir modelos de conducta.

Antes estas nuevas expectativas y centrándonos en el hecho que nos ocupa en este proyecto, la integración y la inclusión social sin ambages, nos planteamos si la sociedad actual está uniendo más a las personas o por el contrario las está separando. Actualmente se habla de la exclusión social como uno de los problemas más 
importantes de la sociedad en la que vivimos. Sin embargo, es necesario manifestar que la exclusión ha existido siempre y la propia sociedad cuando ha generado prácticas educativas ha ido estableciendo parámetros que fomentaban la exclusión de tal manera que ésta se ha convertido en un grave problema en nuestro mundo actual, que aunque se manifiesta en el ámbito educativo, familiar e institucional, es fundamentalmente un problema de índole social. Así lo pone de manifiesto Walker (1997: 8) al definir la exclusión social:

"...se refiere a un proceso dinámico que lleva a ser expulsado, total o parcialmente, de cualquiera de los sistemas social, económico, político y cultural que determinan la integración de una persona en la sociedad. La exclusión social también puede ser vista como una negación (no realización) de los derechos civiles, políticos y sociales de los ciudadanos".

Obviamente este proceso de exclusión se ha manifestado, en mayor o menor medida, en los cuatro modos de acceder a la experiencia sobre el universo y sobre nosotros mismos que hemos descrito con anterioridad: la experiencia directa, la que se obtiene a través de estructuras relacionales interpersonales, la adquirida mediante la lectura y la escritura y la que nos proporcionan las nuevas tecnologías de la información y la comunicación. Siempre ha habido, a nivel social, escisiones entre personas individualmente consideradas y entre comunidades, lo que ha generado clases, mayorías y minorías. El problema actual es que, desde la perspectiva de la pretendida globalización económica y cultural, los excluidos se están convirtiendo en una mayoría preocupante, acrecentándose las desigualdades de manera vertiginosa. Diferentes autores (Castell, 1997; Flecha, 1997; Flecha y Tortajada, 1999; Tedesco, 1995) han intentado buscar una explicación al aumento de la exclusión social en el mundo actual y coinciden en que la causa puede estar en "la nueva sociedad que emerge como consecuencia de un cambio cualitativo en la experiencia humana y la desestabilización que están sufriendo sus fundamentos ante realidades nuevas y ante problemas nunca resueltos" (Gimeno, 2001: 152). Desde esta perspectiva global, la sociedad actual tiene unas características que ayudan a fomentar procesos de exclusión. Parrilla (2000: 27) ha identificado una serie de características básicas de la sociedad actual que configuran el perfil de la misma: es una "sociedad basada en la economía global y la información, es plural y repleta de incertidumbres y contradicciones".

Desde nuestra perspectiva consideramos, de acuerdo con Gimeno (2001), que existen en la actualidad dos debates esenciales con incidencia en la educación. El primero hace referencia a la globalización de la cultura que puede llevarnos a la "homogeneización cultural", lo que puede implicar la exclusión de los menos favorecidos. El segundo tiene como eje nuclear una nueva concepción de la cultura capaz de ser sensible y responder a las diferencias culturales aún a costa de caer en "aislamientos y particularismos que pueden anular la universalidad del individuo en las sociedades complejas" (Gimeno, 2001: 76).

Desde el concepto de globalización que desarrolla alianzas, conexiones, intercambios económicos entre países, entre las formas de vida de los individuos, sus hábitos y sus costumbres, estamos entrando en una nueva forma de concebir el 
mundo que trasciende fronteras en otros tiempos imaginarias. Esta situación aumenta su impacto con la incorporación de las nuevas tecnologías que como afirma Castell (1997: 14) "prometen expandir logros positivos, progreso de la humanidad para todos". Sin embargo en un mundo como el actual, con fuertes desigualdades entre individuos, entre pueblos, entre naciones, la globalización afecta a la identidad de los sujetos ya que todos no ocupamos el mismo lugar en la sociedad y en la cultura y puede generar procesos asimétricos, generando exclusión y desigualdades en aquellos que no tiene posibilidades de acceder a la red económica, política y cultural globalizada, así como desequilibrios sociales importantes. No olvidemos que el $85 \%$ de los bienes del mundo es propiedad de un $20 \%$ de la población. Ortega (2000: 94) describe estas asimetrías ligadas a la globalización y las clasifica en:

a) Asimetrías sociales y educativas. Referidas a la posición dada en una red. Fuera de las redes quedan muchas personas, países y continentes. Por tanto, la inclusión/exclusión es una consecuencia de esa posición en la red. La educación ha iniciado ya grandes cambios facilitando el acceso a través de redes virtuales y nuevos accesos a la información mientras que en algunos países persiste el analfabetismo, el absentismo escolar y la falta de recursos para la educación.

b) Asimetrías en el ejercicio de los derechos humanos. La exclusión en términos de no reconocimiento y violación de los derechos humanos es algo que podemos ver a diario en los medios de comunicación.

c) Asimetrías en el acceso a las tecnologías de la información y en el ejercicio de la libertad de expresión. Sin duda no todos los países ni todas las personas tendrán las mismas oportunidades de acceso a la información generándose una fractura entre países desarrollados y pobres. Pero además como afirma Castell (1997: 18) el acceso a la información no será la única traba sino que la capacidad para procesarla será un nuevo obstáculo que agudizará las diferencias y que a la vez será un reto para la educación en el futuro.

Indudablemente, como afirma Gimeno (2001), para situarnos en este reciente escenario necesitamos de nuevas estructuras de pensamiento que nos permitan encontrar la forma de adaptarnos a él y explorar sus fortalezas y sus debilidades, sus amenazas y sus oportunidades, pero sobre todo estar preparados para experimentar el drama de una globalización que excluye. En este sentido, desde la educación es necesario plantear criterios de igualdad de oportunidades desde políticas educativas que hagan compatibles las diferencias en la escuela, en una escuela inclusiva, para todos. Criterios que ineludiblemente deberán hacer referencia a los derechos de las personas desde una posición de reconocimiento de la autonomía, la libertad y la identidad de todo individuo como ser social y en oposición a la dependencia y la ayuda tal como se han venido preconizando desde los discursos legalistas, médicos y filantrópicos. Barton (1993) se refiere a estas políticas de igualdad de oportunidades como políticas emancipatorias al interpretarlas "como elementos que tratan de devolver las oportunidades a la voz de las personas que habían sido privadas de ellas". "Una política de emancipación se basa en la eliminación o reducción de la 
explotación, la desigualdad y la opresión y promueve la justicia, la igualdad y la participación" (Barton, 1993: 243).

Sin embargo, no debemos olvidar que para generar las condiciones de igualdad las instituciones educativas no se bastan por sí solas, aun cuando reconozcamos que les corresponde un papel preponderante -es evidente, indica Gimeno (2001: 160) que "la educación es condición para la inclusión social"-, sino que es necesario reivindicar nuevas políticas sociales y económicas, sensibles al reconocimiento de los derechos y al fomento de espacios de expresión de las personas, de su libertad de su autonomía y de sus propias capacidades; es necesario reivindicar una calidad en la educación, en la enseñanza (Santos Guerra, 2003, Gairín y Casas, 2003).

\section{LA EDUCACIÓN COMO PROCESO DE SOCIALIZACIÓN: UN PROCESO RELACIONAL Y DE CONSTRUCCIÓN DE UNA IDENTIDAD PROPIA}

Los humanos tenemos una dimensión esencial que es la sociabilidad, como ya afirmara Aristóteles. Nacemos dentro de ciertos grupos sociales que van a ser algo así como el molde en que nos vamos a desarrollar: nacemos en una familia dada, en un barrio determinado, en una ciudad y no en otra, en un país, etc. Y es en el interior de esos grupos en los que vamos a desarrollar nuestra identidad. Probablemente somos más un producto social y cultural que natural. ¿Nos hemos parado a pensar que el nacer en un sitio o en otro es puro azar? ¿Nos podemos imaginar que hubiera ocurrido de haber nacido en otro país u otra familia? Así pues, la identidad personal, el quién soy, se va construyendo en la sociedad de una manera que es como una interacción o, mejor, una dialéctica, entre presiones diferentes basadas en la singularidad de cada uno, su identidad personal, y las presiones del contexto/molde, las del entorno social. Al final todos alcanzamos una identidad social, fruto de esta dialéctica. Después de todo, los seres humanos nacemos como seres asociales, como decía $M$. Weber, y es en el seno de la sociedad que nos convertimos en sociales. Algo que se produce mediante la socialización.

Se suele distinguir en la sociología entre diferentes formas de agrupación social, al menos entre estas:

a) Masa: es nivel menor de interacción y comunicación; en ella nos perdemos y somos como un número (la imagen de un rebaño puede ayudar a entenderla).

b) Comunidad de intereses: la unión aquí se basa en los intereses compartidos por unos individuos. Se da un nivel de co-responsabilidad y de objetivos (una ONG o federación deportiva, son ejemplos).

c) Sociedad: se comparten intereses pero no objetivos o actividad. La vinculación lo es por contrato, base de las normas o reglas sociales y jurídicas que regulan nuestra pertenencia.

d) Comunidad de vida: concepto muy reciente y acertado que supone el nivel de sociabilidad más alto pero respetando la libertad y basado en la tolerancia, ya que su pertenencia es voluntaria. Se comparte simplemente las ganas de querer vivir, sentir y pensar juntos. La confianza mutua y la amistad son su base. 
El modo en que nos integramos y adquirimos una identidad social es la socialización. No olvidemos que nacemos asociales, y dentro de la sociedad adquirimos hábitos de conducta, normas y reglas de comportamiento que nos convierten en seres sociales. La socialización nos introduce en la sociedad y el peaje que pagamos por ello es la adopción de sus formas y normas de conducta: leyes jurídicas, reglas morales, normas de convivencia, etc. En este sentido, se suele distinguir entre dos tipos de socialización, la socialización primaria y la socialización secundaria.

La socialización primaria se realiza a través de aquellos espacios sociales o moldes, Ilamados agentes sociales, como la familia, la escuela o el estado. Durante la infancia, los niños se apropian de los roles, actitudes y valores de las personas que les importan (padres, hermanos, maestros, amigos) y se identifica con ellos. Es el primer paso hacia la identidad. Los niños no sólo aceptan los papeles sociales (roles) de estas personas sino el mundo que representan. La socialización implica la interiorización de roles. Un proceso que va desde lo concreto a lo general. El proceso de socialización primaria es más que un acto cognitivo, es vivencial y emocional, es la base de la identidad. Es la adquisición de normas y valores de su cultura. De este modo la socialización convierte a un individuo en un ser social.

La socialización secundaria es un proceso mediante el que se internalizan (interiorizan) submundos o realidades distintas al mundo base, fundamento de la socialización primaria. Es una segunda socialización realizada a través de instituciones políticas, religiosas, laborales, etc. A diferencia de la socialización primaria, donde el niño no puede elegir, ya que los patrones socializantes le vienen impuestos, en esta sí puede elegir los patrones y pautas que interiorizará o hará suyos. Hay una menor carga emocional y se relaciona con la madurez. Pueden aparecer conflictos de identidad y coherencia con la socialización primaria al descubrir que la realidad paterna o familiar no es la única ni la mejor, surgiendo problemas de identificación con la familia. Resulta esclarecedor el texto que recogemos de Berger y Luckmann (1984: 24).

"El individuo no nace miembro de una sociedad: nace con una predisposición hacia la socialidad, y luego llega a ser miembro de una sociedad. En la socialización primaria no existe ningún problema de identificación, ninguna elección de otros semejantes. La sociedad presenta al candidato a la socialización ante un grupo predefinido de otros semejantes a los que debe aceptar en cuanto tales, sin posibilidad de optar por otro arreglo. Hay que aceptar a los padres que el destino nos ha deparado. Esta desventaja injusta inherente a la situación de hijo tiene la consecuencia obvia de que, aunque el niño no sea un simple espectador pasivo en el proceso de su socialización, son los adultos quienes disponen las reglas del juego. El niño puede intervenir en el juego con entusiasmo o con hosca resistencia, pero por desgracia no existe ningún otro juego a mano. Esto tiene un corolario importante. Como el niño no interviene en la elección de sus otros significantes, se identifica con ellos casi automáticamente. El niño no internaliza el mundo de sus otros significantes como uno de los tantos mundo posibles, lo internaliza como el mundo, el único que existe y que se puede concebir, (...) Por esta razón, el mundo internalizado en la socialización primaria se implanta en la conciencia con mucha más firmeza que los mundos internalizados en socializaciones secundarias". 
Queda claro, por tanto, el hecho de que los seres humanos por naturaleza tienden a relacionarse unos con otros. Esta característica se expresa en el proceso de socialización que interiorizan con el transcurso del tiempo a través de contactos, relaciones e interdependencias, creando comunidades diversas a las que sienten que pertenecen. En ellas se generan a su vez procesos de acercamiento y afinidad entre los sujetos a la vez que distanciamientos, cuestiones ambas propias de la convivencia humana.

Las relaciones entre los individuos y los vínculos que se establecen entre ellos han de ser compatibles con la libertad y la autonomía propia de cada individualidad. ¿Cuál es papel de la educación en este proceso relacional y de construcción de una identidad propia? Gimeno (2001: 107) se manifiesta al respecto indicando que el papel de la educación será el de "contribuir a asentar y fundamentar esas dos dimensiones aparentemente contradictorias convirtiéndose en un instrumento para la conquista de la autonomía y de la libertad, a la vez que fomentar el establecimiento de lazos sociales para la convivencia pacífica".

En este sentido, nos vamos a remitir a la conceptualización que Pérez Gómez (1992: 17), desde una perspectiva global y diacrónica, atribuye a la educación como proceso de socialización:

"Desde que la configuración social de la especie se convierte en un factor decisivo de la hominización y en especial de la humanización del hombre, la educación en un sentido amplio cumple la ineludible función de la socialización". "... A este proceso de adquisición por parte de las nuevas generaciones de las conquistas sociales, a este proceso de socialización, suele denominarse genéricamente como proceso de educación".

Esta socialización es entendida como un proceso dirigido a la integración de las personas en la sociedad de cara a conseguir su participación en la cultura.

Como podemos observar, esta concepción de Pérez Gómez está muy relacionada con la conformación y desarrollo del carácter social del grupo humano como especie, y con la pervivencia y transmisión de los logros conseguidos a las generaciones venideras. Tiene, por tanto, un marcado carácter cultural. En todas las sociedades, grupos y culturas se dan y se organizan procesos y experiencias de aprendizaje (parcialmente conscientes y parcialmente inconscientes) a través de los cuales la generación de más edad incita, induce y obliga a la generación más joven a adoptar las formas de pensar, sentir y obrar tradicionales. El papel adjudicado a la Educación sería el de organizar el proceso de adquisición de la cultura que facilite la integración del individuo en la sociedad.

El problema estriba en que las formas concretas que se pueden establecer desde el ámbito educativo para conseguir dicha integración y participación social, pueden variar y divergir ampliamente. Sin embargo, para el nivel de análisis que queremos establecer podemos convenir que, de manera general y aceptando las diversas fórmulas aplicadas, la acción pedagógica tiende a preparar al hombre para la vida en sociedad y el acceso a la cultura, entendiendo ésta en su sentido amplio (socio-antropológico) y no restringido que la relaciona con un determinado tipo de conocimiento y manifestaciones artísticas. Algunos y algunas han ido descubrien- 
do la inclusión, su cultura, política y prácticas al enfrentarse a su ejercicio profesional sumergido en el ámbito de la atención a la diversidad (Porras, R.; García, M. y Cotrina, M. 2009).

Por otra parte, la educación como socialización no puede entenderse al margen de los valores ideológicos, económicos y políticos de la sociedad (Lundgren, 1992). En este sentido, la educación entendida como participación cultural cobra diferentes matices según el paradigma imperante en la sociedad. De acuerdo con estos planteamientos, lo que podemos entender por cultura y participación social puede tomar también diferentes sentidos, tales como la cultura como algo objetivo y neutral (positivismo) o la cultura como recreación, al ser reinterpretada y negociada por sus participantes (Bruner, 1988, 1997). La educación, por tanto, orienta nuestra identidad social. Desde esta perspectiva el desarrollo de formas dominantes de sociabilidad están determinadas de alguna manera por la cultura que canaliza la configuración de relaciones interdependientes entre los individuos. En este sentido Gimeno (2001: 110) manifiesta que "las relaciones sociales tienen que ver con el componente cultural que proporciona los significados que las desencadenan, las hacen posibles, las nutren, las facilitan o las dificultan".

Parsons (1982), asimilando en su sistema social parte sustancial de las ideas de Durkheim, ve en la educación (en su terminología presentada como socialización) el mecanismo básico para la constitución de sistemas sociales y de mantenimiento y perpetuación de los mismos en forma de sociedades. Sin la socialización el sistema es incapaz de mantenerse integrado, preservar su orden, su equilibrio y conservar sus límites. Para que el sistema sobreviva, los nuevos individuos que en él ingresan necesitan asimilar e interiorizar los valores y las normas que rigen su funcionamiento (Carbonell i París, 2003). Parsons, al contrario de Durkheim, no resalta tanto el aspecto coercitivo del sistema frente al individuo, pero señala la complementariedad de los mecanismos que actúan con el fin de satisfacer los requisitos del sistema social y del sistema de personalidad. Así como el sistema tiene necesidad de socializar sus miembros integrantes, también el individuo tiene necesidades que solamente el sistema puede satisfacer.

Sin embargo, aún cuando ciertos autores asuman que los procesos de socialización, incluso en las escuelas, no son unidireccionales, sino que resultan de una interacción entre el individuo y otras dimensiones de su experiencia social, los investigadores están más dispuestos a entender y ubicar los resultados del proceso como una trayectoria psicosocial en el ámbito individual, y no considerar aspectos de la dinámica entre instituciones y grupos que repercuten en la escuela y redefinen la socialización.

Cuando algunos investigadores se distancian o rechazan la perspectiva de una autonomía por parte de los sistemas educativos hacia la sociedad -asumiendo que hay una determinación entre estas instancias, y que ésta refleja las situaciones de segmentación y conflicto que viven internamente las sociedades y no una homogeneidad, lo cual también incluye considerar culturas políticas diferenciadas para las cuales la educación cumple distintos papeles de socialización-, entramos entonces en un espacio de reflexión, donde la educación es vista como el mecanismo reproductor por excelencia de la vida social, su jerarquía y sus desigualdades, y a partir 
del cual no se operan cambios estructurales en la sociedad. Tales teorías introducen los conceptos de poder y dominación en el análisis de la relación educación y sociedad, compartiendo percepciones fundamentalmente diferentes de cómo el poder y el control funcionan en el interés de la sociedad dominante, tanto dentro como fuera de las escuelas (Giroux, 1986: 107).

En la década de los setenta este tipo de acercamiento produjo muchos análisis sobre el sistema de enseñanza. Sus aportes buscan demostrar la imposibilidad de la educación para construir sujetos forjadores de su acción, pues apenas hay lugar para la formación de agentes estructuralmente determinados. Muy escuetamente diríamos que los estudios referidos pertenecen a la perspectiva estructuralista, donde se asume la sociedad como un todo no homogéneo, más bien de heterogeneidades jerarquizadas, como las tres instancias de prácticas sociales: económica, política e ideológica, donde la primera es la fundamental para la estructuración de la sociedad en clases, situación que se origina en la división social del trabajo y la apropiación diferencial de los medios de producción. La relación entre clases es de carácter antagónico y se subsume a esquemas de dominación y sujeción que atraviesan las tres instancias de prácticas sociales.

A partir de esos supuestos se establece la preocupación de señalar y comprender los mecanismos sociales que permiten la continuidad y conservación de tales estructuras de dominación. Gran parte de los autores encuentran en la educación formal la institución que garantiza en parte la reproducción de la sociedad y la aceptación de las desigualdades.

Sin embargo, el sistema educativo refleja intereses de dominación y se propone operar como "transmisor de representaciones y prácticas que facilitan la legitimación y reproducción de intereses dominantes en un orden social" (Giroux, 1986: 207). Esta configuración de representaciones es, en el contexto estructuralista, lo que se considera la ideología dominante. En tal caso, diríamos que la escuela es por antonomasia una agencia de socialización, unilateralmente determinada por los grupos dominantes. En la escuela se conocen y se aceptan imágenes, conceptos y contenidos como elementos objetivos, externos, los cuales son apropiados por los individuos y regulan sus propias interacciones. Es interesante señalar que en esta corriente la socialización, por medio de la escuela, se contempla prácticamente sólo la transmisión de la ideología dominante, pues cuando se acepta la existencia de ideologías vinculadas a otros grupos sociales, con cuya diseminación se compite, se considera que éstas se propagan por otros medios y no por la escuela. El estructuralismo no rechaza la posibilidad de que se viva en las instituciones escolares alguna autonomía, la cual puede incluir reflejos de la vida social conflictiva y antagónica que se da en su exterior, pero esto no es suficiente para penetrar la escuela y producir cambios estructurales. Su función es todo lo contrario, "es de manutención" (Giroux, 1986: 113).

Por otra parte, es necesario destacar la existencia de algunos autores que, aceptando la visión estructuralista para el entendimiento de la vida social, se interesan sobre todo por explicar los mecanismos de reproducción en el ámbito cultural, tanto en sus espacios grupales como no grupales. Es el caso de los trabajos de Bordieu y Passeron (1977) y Bernstein (1977). Entendemos que el esfuerzo que realizan se 
adhiere en el objetivo de rebasar la idea de la función escolar como adoctrinamiento o inculcación ideológica, proponiendo el campo de la transmisión cultural y la socialización como el idóneo para encontrar los elementos que subyacen a la estructura, y que operan como mediadores en este proceso. En ellos está presente la idea de una sociedad desigual, en la que operan conflictos y una estructura de dominación que se reproduce por varios mecanismos, siendo la educación uno de ellos, aun cuando ésta puede mostrar cierta autonomía.

En tales estudios se sostiene que la reproducción cultural engloba aspectos políticos y la socialización que se da en las instituciones escolares es reconocida y considerada de interés para el estudio de la dinámica social. Develar estos mecanismos es el principio del trabajo de esos investigadores, que finalmente están interesados en el análisis de la permanencia de una cultura de clase que es dominante. A la importancia de esas aportaciones correspondió gran número de esfuerzos posteriores que se apoyan en sus teorías para analizar casos específicos de situaciones educativas.

Por otro lado, estos autores expresan que la socialización no se hace solamente en términos de infundir ideas, conceptos u opiniones, sino que además se infunden actitudes y disposiciones que regulan los comportamientos. Estos principios estructurados que organizan pensamiento y acción son los habitus y son transferibles e inconscientes, además de comunes para determinados grupos.

Siguiendo el esquema de Bourdieu y Passeron, diríamos que estamos en una situación de imposición ideológica en lo individual y de conformación de capital cultural en lo grupal, que tiende a la homogeneidad y no a rupturas y cambios. La socialización produciría resultados inevitables y la cultura dependería de un esquema estructurado de disposiciones y actitudes que es inexorablemente transmitido a los miembros de una sociedad. Así estos autores complementan el esquema de reproducción social añadiendo y dilucidando el aspecto de la reproducción cultural, pero siempre en un proceso con profundas conexiones o determinaciones respecto a la estructura de reparto del capital económico y de participación del poder (Pérez Gómez, 1991).

El interés en comprender las cuestiones relacionadas con la persistencia de estructuras de poder y control a partir de la transmisión de pautas culturales fue también una temática de la que se preocupó Basil Bernstein. Sus trabajos se desarrollan dentro de una perspectiva sociolingüística y, en el contexto de procesos de socialización, busca "deslindar aquellos que operan en la distribución social del conocimiento, que es desigual y está afectada por el sistema de clases" (Bernstein, 1991: 288).

Al dar continuidad a nuestra intención de relacionar las posturas respecto a la educación y su correspondiente derivación de características para la socialización, podríamos señalar entonces que Bernstein, a pesar de no trabajar con un esquema mecanicista de correspondencia estructural entre clase, escuela y control político, deja poco espacio para que la educación pueda operar como un agente que amplíe las delimitaciones simbólicas definidas por las relaciones de clase y establezca formas más amplias de participación, que logren sobrepasar los mecanismos de reproducción social. En este caso se podría pensar que la educación formal mantendría en compartimentos estancos las distintas culturas de los diversos grupos sociales, fortaleciendo el predominio de la cultura de los grupos jerárquicamente altos. Si 
transferimos este razonamiento al ámbito de la cultura y de los procesos de socialización, cobra sentido decir, según estos autores, que el proceso escolar resultaría en la reafirmación de una cultura dominante y la inhibición o devaluación de la cultura de los grupos sumisos.

Es claro que las explicaciones culturalistas proponen una noción de cultura como experiencia vivenciada, que intenta recuperar la acción humana. Pero lo hace al costo de "devaluar la importancia de las prácticas materiales y los diferentes niveles de especificidad que ellas tienen dentro de la totalidad social" (Giroux, 1986: 179). Si bien un culturalismo a "ultranza" no resuelve el problema del dualismo acción-estructura, la idea de la cultura rescata al "sujeto" y abre perspectivas interesantes para el análisis de la cultura y de la socialización en los procesos educativos formales.

Cuando se entiende la cultura como una creación que supone la determinación y también la acción humana innovadora, que se traduce tanto en prácticas reproductivas o transformadoras, permitiéndonos considerar la existencia no sólo de clases sociales, sino también de otros grupos que participan en procesos de creación, resistencia o sumisión cultural, produciendo significados y acciones específicas, revelando la cultura como un armazón heterogéneo de prácticas y creencias, se vuelve más complejo el esquema interpretativo de los procesos de dominación de culturas de clases. Por otro lado al señalar que eso es así, percibimos que a esa heterogeneidad de prácticas se corresponde una heterogeneidad de actores sociales.

No es posible suponer que las relaciones sociales de producción sean la única fuente de la dinámica social, aun cuando no se puede dudar de su centralidad dada su capacidad de alterar la base material de la sociedad. Junto a esta dinámica económica encontramos que lo social, lo político y lo cultural también poseen una autonomía relativa frente a los demás y es justamente esa autonomía relativa la que posibilita la interdependencia. Este argumento nos permite pensar que los sectores sociales no clasistas se ubican en posiciones sociales, políticas y culturales cuyas dinámicas y oposiciones le son propias. Lo que importa es tener en cuenta que la dinámica de los sujetos sociales clasistas está entrelazada con la dinámica de otros sujetos sociales que se organizan y actúan en oposiciones no económicas. En consecuencia, resulta artificial y arbitrario reducir la dinámica a un solo tipo de sujetos sociales. Por el contrario, la interrelación entre las esferas se expresa como mediaciones que determinan la especificidad de un sujeto social, cualquiera que sea su origen. En este sentido no hay sujetos puros, que estén anclados y determinados por el acontecer de un solo plano; "el sujeto siempre estará determinado por mediaciones sociales, económicas, políticas y culturales" (Durand Ponte, 1989: 35).

En un primer momento es claro que entre los elementos que propician esta heterogeneidad estarían la división sexual y social del trabajo, diferencias raciales y aun la variedad de las instituciones sociales que son específicas y autocontenidas en muchos casos, pero que mantienen relaciones entre sí. Así, las realidades experimentadas por los individuos poseen una complejidad que no se agota en un esquema de clase, y la cultura de los grupos supera esta situación y se desarrolla en una tesitura de tensiones de diversas índoles. Acorde con esta visión se asume que el sujeto social se reconceptualiza. Es un sujeto multideterminado, que ya no está ins- 
crito en una lógica única de determinación y dominación, y presenta sin duda características que le confieren la oportunidad de la reflexividad.

Hasta ahora hemos presentado una visión de la estructura como determinante de la acción de los individuos o de los sujetos sociales y si bien, como hemos afirmado, la estructura cambia por la acción individual o de los sujetos, es del todo insuficiente para entender a los sujetos o a los individuos como entes que poseen alguna autonomía y voluntad propia. Para reivindicar esta autonomía es indispensable introducir el concepto de reflexibilidad, es decir, la capacidad de los individuos de pensar su acción, sus prácticas e incluso las reglas y, a partir de ello, "reaccionar críticamente, proponer nuevos principios clasificatorios y luchar por ellos" (Durand Ponte, 1989: 25).

Esta forma de ver la cultura y los sujetos se encuentra hoy muy cerca de los sociólogos que están preocupados en estudiar los mecanismos reales y cotidianos de la reproducción cultural en las escuelas. Si estamos interesados en la socialización a partir de la educación y referida a una determinada cultura, estas posturas recientes son importantes para reelaborar una perspectiva de análisis sobre estos temas. Siguiendo estos razonamientos, tendríamos que aceptar las consecuencias de la multideterminación y la reflexividad en los sujetos sociales, y en la dinámica social y sus movimientos.

Al considerar estos elementos observaríamos una institución escolar que vive y refleja esta realidad de tensión y movimiento entre sujetos sociales, y como tal no sólo representa un sitio de reproducción sociocultural de desigualdades, sino también de intereses emancipatorios, tendentes quizá a la equidad. En conclusión, la escuela revelaría una lucha cultural entre sujetos determinados por elementos como clase, raza, género y religión. En estas interrelaciones se vive y se constituye la cultura y los sujetos en razón de sus experiencias mediadas por instancias institucionales y normativas que con frecuencia atienden a lógicas de dominación, aunque no es exclusivamente así.

En estos términos no hay neutralidad de la escuela con relación a esos enfrentamientos de sujetos distintos. Hay oposiciones, imposiciones, resistencias culturales que ejercen los diferentes sujetos en el espacio educativo dado y los resultados de esa interrelación, aun cuando en general funcionen a favor de la reproducción y admitan también salidas transformadoras, que son dependientes del tipo de mediación que se plantee. Son posibilidades a explorar por los actores envueltos en el proceso y otros agentes que traen una visión de cambio de otras esferas públicas alternativas.

La existencia de sujetos multidimensionados hace que consideremos nuevas y complejas relaciones sociales que sólo se resuelven en presencia de valores democráticos, los cuales admiten la particularización de sus derechos y su defensa. La idea de un sujeto universal, abstracto, del que emanan derechos, se abandona y deviene una perspectiva donde se reconocen diferencias, se particularizan los sujetos y se apoya la defensa de las múltiples posiciones de los mismos. Las identidades no son permanentes, son fuertemente indeterminadas y diferenciadas, y casi siempre conflictivas. Desde una perspectiva democrática, múltiple y plural, en la cual los derechos democráticos individuales sólo adquieren sentido cuando son ejercidos y reconocidos colectiva- 
mente, es donde se conforma el sujeto social no unitario, o sea una forma de individualidad "verdaderamente plural y democrática" (Mouffé, 1994: 93).

Parece que la mayoría de los autores que hemos revisado (y faltarían muchos otros) razonan a partir de modelos de sociedad considerados políticamente avanzados, en la medida que pueden ser caracterizados como democracias estables y modernas, con niveles aceptables de participación ciudadana y de más equidad en la distribución de recursos económicos. Aún así, las transformaciones sociales, la diferenciación y complejidad de las relaciones entre los individuos, están marcando una nueva perspectiva de análisis de la vida social y sus actores, y consecuentemente de las demandas que una sociedad compleja puede hacer respecto a la formación ciudadana y la cultura política.

En este contexto teórico hay autores que han trabajado con el objetivo de definir y discutir el papel de la escuela y de la educación en procesos de socialización. Estas aportaciones son respuestas a las demandas crecientes de formación de nuevas individualidades particulares y plurales para la convivencia en esos nuevos espacios políticos. Ciertamente nos encontramos en un proceso de intensos cambios culturales y nuevas orientaciones en las pautas de comportamiento. Se afirma que los procesos de transición social se basarán en la acción humana, en parte definida individualmente, distanciada de factores estructurales pero vinculada a identidades culturales concreta que convivirían articuladas en términos de su capacidad de comunicación y avance.

Gran parte de estos autores plantean que la educación, en su acepción más amplia, tendrá que ser la responsable de esta formación, pero deberá enfrentarse con una "escuela tradicional que por inercia u oposición no está respondiendo a nuevas demandas ni desempeñando las funciones nuevas que ahora son necesarias" (Giroux, 1986: 309). Además, esta reformulación se fundamenta en una nueva definición de objetivos de la educación. Los profesores, tomados como mediadores de los procesos educativos, estarán comprometidos con las transformaciones democráticas e interesados en la construcción de una ciudadanía plural, que ya se encuentra en otros espacios públicos alternativos. En este caso la escuela asumiría claramente un papel socializador y reflejaría y reinventaría una nueva sociabilidad, donde los esquemas deterministas de reproducción social podrían quedar rebasados.

Por otra aparte, los educadores necesitan entender que las diferentes identidades entre los jóvenes se están produciendo en nuevos espacios generalmente ignorados por las escuelas. La pedagogía debe redefinir su relación con las formas modernas de cultura, privilegio y regulación normativa y servir como vehículo de interpretación y potenciación mutua. La pedagogía como práctica cultural crítica necesita abrir nuevos espacios institucionales en los que los estudiantes puedan experimentar y definir qué significa ser productores culturales. En este caso, las escuelas pueden ser repensadas como espacios públicos, como zonas fronterizas de cruce comprometidas activamente en producir nuevas formas de comunidad democrática organizadas como lugares de interpretación, negociación y resistencia.

Con estas posturas y exigencias frente a la escuela es claro que actualmente su función de agencia socializadora política no puede ser soslayada y se enmarca con mucho vigor en sociedades altamente complejas. A esto se debe la preocupación y 
la insistencia en la discusión de temas sobre ética, valores y educación pluricultural que se han generado en el campo educativo en los tiempos actuales. Para finalizar manifestar que parece claro que las instituciones educativas no son vistas hoy como precondición, en sí mismas, de la construcción o cambio en las formas de participación y actitudes sociales, sino más bien son integrantes de un complejo proceso de constitución de la vida social. Es conveniente no olvidar este planteamiento para que no se reincida en expectativas desmesuradas y voluntaristas respecto a los procesos educativos, en cuanto generadores de cambios sociopolíticos, como también en un desperdicio de los mismos en términos de coadyuvar tales cambios. La educación debería promover estilos de vida que impliquen demandas de participación y exigencias crecientes. La escuela para todos, no aislada, que refleja la vivencia amplia de sus actores, constituidos en una pluralidad de sujetos sociales, podría ser resultante de esta propuesta de escuela y sus tareas inseparables de socialización y formación para el acceso al mundo laboral. Bajo este concepto la educación establecería las relaciones adecuadas con otros espacios de actividad, y se constituiría en elemento central para el desarrollo social, abandonando propuestas aisladas y voluntaristas.

\section{DiverSIDAD, DIFERENCIA Y DESIGUALDAD}

Toda organización educativa se configura en torno a una comunidad en la que se integran alumnos, profesores y padres y todos ellos, como miembros de la comunidad educativa, son diversos y suelen proceder de diversas culturas, presentar necesidades, o, simplemente, ser distintos en cuanto a concepciones sobre la educación, intereses, ritmos y estilos de aprendizaje, motivaciones y expectativas.

"Por condicionamientos diversos, podemos llegar a ver como anómalo lo que son condiciones normales de la realidad. Es un hecho empírico comprobable desde el sentido común, antes de ser una constatación del pensamiento científico, que, desde el punto de vista biológico, psicológico, social y cultural, los seres humanos diferimos unos de otros. Cada uno constituimos una radical individualidad al lado de otras tan singulares como la nuestra. La particular dotación con la que venimos al mundo, el uso que hacemos de nuestras cualidades, la singularidad de los contextos por los que transitamos, la peculiar asimilación que hacemos de esos contextos y las iniciativas individuales que tomamos, hacen de cada uno una persona con una biografía y una idiosincrasia singulares" (Gimeno, 1999: 68).

El problema es que durante muchas décadas, como hemos podido comprobar en los epígrafes anteriores, nuestra sociedad se ha preocupado por conocer cuáles son las pautas más habituales y comunes en todos los aspectos de nuestra vida (características físicas, capacidades, hábitos...). En nuestra cultura, con frecuencia, lo que más se valora de las personas no es lo que son, sino su grado de aproximación a los patrones considerados ideales. La cultura de la norma crea, así, unas expectativas iguales para todos, que son el referente para valorar las formas de ser y los progresos de las personas. Se fomenta así una cultura de la homogeneidad en la que vivimos inmersos, donde la "diferencia aparece como algo no deseable, y constituye, por tanto, un problema" (Del Carmen, 2000: 7). 
En numerosas ocasiones, cuando se habla de diversidad en el aula, se piensa en alumnos y alumnas con dificultades. A nivel social se piensa de igual manera. Hablar de diversidad es hacer referencia a personas que "están fuera de la norma" y así se ha puesto de manifiesto a lo largo de la historia mediante mecanismos de segregación y exclusión. Es esclarecedora la cita de Ainscow (2001:25)

"Conviene recordar que las escuelas, como otras instituciones sociales, están influidas por las percepciones de la categoría socioeconómica, la raza, el idioma y el género. Es esencial, por tanto, preguntarse cómo influyen esas percepciones en las interacciones de clase. De este modo, hay que extender la insistencia actual en los métodos hasta revelar la visión deficitaria, profundamente enraizada, de la 'diferencia', que define a ciertos tipos de alumnos como individuos 'carentes de algo'".

La atención a la diversidad debería plantearse como algo relacionado con todo el alumnado, más aún, con toda la comunidad educativa. Es una transversal permanente de todo quehacer educativo. En este sentido, Gimeno (1999: 67) manifiesta:

"Es un aspecto transversal en el pensamiento y en la investigación. Por otro lado, en este tema nos vemos obligados a transitar por el filo de una extraordinaria ambigüedad al utilizar el lenguaje. La diversidad alude a la circunstancia de los sujetos de ser distintos y diferentes (algo que en una sociedad tolerante, liberal y democrática es digno de ser respetado). Aunque también hace alusión, por otra parte, a que la diferencia (no siempre neutra) sea, en realidad, desigualdad, en la medida en que las singularidades de sujetos o de grupos les permitan a éstos alcanzar determinados objetivos en las escuelas y fuera de ellas en desigual medida".

Pero diversidad no es lo mismo que desigualdad. La confusión entre los conceptos de diversidad y desigualdad muestra la ambigüedad existente en el debate y en la práctica de la educación en la diversidad. Así, cuando algunos autores previenen contra el peligro de introducir bajo la educación en la diversidad el proyecto neoliberal de reproducir las desigualdades sociales, se está equiparando uno y otro concepto, de manera que educar en la diversidad sería sinónimo de dar a cada uno lo que necesita para seguir estando donde está en la jerarquía social y cultural. $O$, en sentido contrario, se argumenta que un exceso de comprensividad puede tener los efectos contrarios, pues al intentar igualar lo imposible se acaba produciendo más desigualdad (Ollero, portavoz del PP, en Cuadernos de Pedagogía, 234, enero 1995).

No creo que exista nadie con talante democrático que no abogue por la igualdad de todos los ciudadanos. Se habla de igualdad de todos ante la ley, de igualdad de oportunidades, de igualdad de derechos. Desde este mismo plano discursivo tendríamos que hablar también de desigualdad ante la ley, desigualdad de oportunidades..., lo cual no es equiparable a pensar sobre la diversidad ante esas mismas situaciones desde el ámbito de las diferencias. Cuando se habla de "igualdad ante..." no se excluyen las diferencias, sino que éstas son las que garantizan la posibilidad de reclamar el derecho de "ser iguales ante...". En este sentido son elocuentes las palabras de Pérez Tapias (1996: 142): 
"Educar para la igualdad es educar para el mestizaje, en el respeto a la alteridad que hace posible el mutuo enriquecimiento desde las diferencias. Si logramos educar en la igualdad que desde el punto de vista de la humanidad interesa, estaremos avanzando por el mejor camino hacia la justicia, el que conduce a la libertad de todos y a que nadie vea frustradas de raíz, por el azar de la naturaleza o por determinada ubicación social sobre la que recae la carga opresiva de la historia, sus posibilidades de autorrealización".

Por tanto, desde nuestra perspectiva, "hablar de una escuela para todos supone adoptar una posición ideológica clara frente al hecho educativo y su función social, rechazando la desigualdad bajo la cual se establecen jerarquías y aceptando la diferencia como un valor positivo" (García e Illán, 1999: 30).

En este sentido, no es lo mismo la diversidad de gustos literarios que la diversidad que se establece entre quien no comprende un texto y quien es capaz de recrearlo. Como tampoco es lo mismo ofertar materias que permitan a los alumnos orientarse que clasificarlos previamente. Si entendemos por diversidad todo aquello que hace a las personas y a los colectivos diferentes, y por desigualdad todo aquello que establece jerarquías en el saber, el poder o la riqueza, el panorama se nos aclara. Ya hemos descrito en anteriores ocasiones (Torres González, 1997, 1998, 1999, 2002) que las manifestaciones de la diversidad en el ámbito de la educación derivan de factores sociales, económicos, culturales, geográficos, étnicos y religiosos, así como de las distintas capacidades intelectuales, psíquicas, sensoriales y motóricas. Podemos observar claramente dos dimensiones de estas manifestaciones: diversidad, entendida como todo aquello que hace a las personas y a los colectivos diferentes por cuanto que la naturaleza humana se revela como múltiple, compleja, diversa y desigualdad como aquellos procesos que establecen jerarquías en el saber, el poder o la riqueza de los individuos o colectivos. La diversidad, por tanto, hace referencia a factores físicos, genéticos, personales y culturales, mientras que la desigualdad hará referencia a factores sociales, económicos y políticos. Si extrapolamos estos conceptos al campo educativo, podremos hablar de personas diversas o diferentes en cuanto a capacidades, estilos y ritmos de aprendizaje, motivaciones, valores culturales y podremos hablar de desigualdades en situaciones que producen desventajas y carencias en relación con otros individuos o colectivos. López Melero (1997: 174), incidiendo en esta misma línea, que compartimos, lo plantea así:

"...deseo aclarar que entiendo por diversidad, qué por diferencia y qué entiendo por desigualdad. La diversidad hace referencia a la cualidad de la persona por la que cada cual es como es y no como nos gustaría que fuera. Este reconocimiento es precisamente lo que configura la dignidad humana. La diferencia es la valoración de la diversidad y es precisamente en esta valoración donde hay varias manifestaciones ya sean de rechazo (antipatía) como de comprensión (simpatía). Es la consideración de la diversidad como valor. Y la desigualdad es el establecer jerarquías entre las personas por criterios de poder social, político y económico. Es precisamente lo contrario de la igualdad".

Así pues, todos y todas somos diferentes (diversos) y todos y todas padecemos de alguna situación de desventaja o carencia, independientemente de que decidamos 
compensarla o no. La acción educativa desde una opción democrática se regirá por dos líneas estratégicas:

- Respetar/compartir las diferencias y

- compensar/superar las desigualdades.

En general seguimos dos grandes modelos para atender de manera integradora la diversidad de alumnos. Un modelo consiste en la profundización de las situaciones de diversidad: se trata de respetar, promover e incluso intensificar los aspectos diferenciadores -valores culturales, intereses y motivaciones, capacidades peculiares... Predominan las estrategias diferenciadoras y centrífugas. El otro modelo es el de la superación de situaciones de diversidad: se trata de igualar, compensar, tender hacia la aproximación de niveles y de situaciones de partida desiguales -ritmos y procesos de desarrollo, desigualdades de origen sexista, desajustes importantes con relación a los objetivos socialmente convenidos, necesidades educativas especiales... Las estrategias homogeneizadoras y centrípetas son las que se corresponden con esta línea de actuación.

Pero la cuestión se nos complica cuando constatamos qué factores de diversidad son generadores de desigualdad, sea por efectos sociales o sea por efecto de la misma escuela: si el registro lingüístico de un chico no se acomoda al estándar de la escuela tiene más problemas para aprender que otros; si se es chica se tienen más problemas para acceder a la cultura tecnológica. En estos ejemplos sucede que la diferencia de código lingüístico en un caso, y la diferencia de género en otro, se convierten en factores de desigualdad, por la jerarquización que producen las prácticas escolares y los valores sociales.

La realidad de las personas y de la actividad educativa es una totalidad compleja y por tanto no es fácil determinar qué debe profundizarse y cuál superarse. Sólo una adecuada combinación de estrategias diferenciadoras y de estrategias igualadoras puede aproximarnos al objetivo de desarrollar en todo el alumnado sus máximas potencialidades. Ello plantea interrogantes diversos sobre el equilibrio entre lo básico y lo diversificado en la articulación de toda organización educativa, sobre los campos de actuación y definición de las estrategias igualadoras y de las diferenciadoras, y sobre la cultura del profesorado que es quien en definitiva toma las decisiones.

Hemos podido evidenciar anteriormente como la sociedad y la educación han reaccionado de forma muy parecida ante la diversidad humana. Tanto la escuela como la sociedad en su perspectiva educadora, han desplegado multitud de acciones para intentar corregir la diversidad, convirtiendo las diferencias entre los sujetos en situaciones claras de desigualdad, en la ansiada búsqueda de un determinado orden social, sometiendo a los individuos a sus normas, a su currículum y a su estructura organizativa (Forquín, 1985). Los que hemos vivido la escuela segregadora y excluyente tanto como alumno como de profesor podemos dar fe de ello. Parece claro pues que los intentos de la institución educativa de afrontar la diversidad se han basado en un intento casi permanente de ordenación y tratamiento diferenciado de la misma (Gimeno, 2000). En este sentido la diversidad ha sido abordada, tanto en la teoría como en la práctica, desde la individualidad, existiendo una tendencia a "clichar" a los individuos en niveles o escalas de clasifica- 
ción, es decir a estructurar los colectivos de personas en grupos diferenciales. Pero no olvidemos que a nivel social ha ocurrido lo mismo. ¿Podemos hablar entonces de una escuela reproductora de las pautas sociales? ¿Podemos decir que la diversidad era algo con visos de universalización? como planteaba Ferrández (1997: 15). Creo que ciertamente sí, entre otras razones porque la sociedad actual es altamente diferencial a la vez que reduccionista a situaciones actitudinales de corto espectro (muchos padres se niegan a que sus hijos compartan la educación con sujetos diferentes: gitanos, portadores de SIDA, magrebies). Por otra parte, se habla en la actualidad de la "socialización pautada gracias a la cual todos tenemos que pensar, sentir, hacer y estar dentro del mismo pentagrama y orquestados en la misma clave de sol" (Ferrández, 1997: 19). Por último, hemos de considerar que la necesidad de un cambio axiológico a nivel social y educativo es imprescindible pero a la vez complejo. Manifiesta Ferrández (1997: 18) que "nada se conseguiría si se produjera un cambio social respecto a la consideración de las personas diversas si a la vez cada persona no reconoce, y menos aún acepta, las características propias de su diversidad". Es un reto social y educativo. La educación, nuestro ámbito de desarrollo profesional, ha de convertirse en la esperanza, en el tesoro, denominado en el informe Delors (1996), que pueda, por una parte, ayudar a educar para una sociedad del futuro más justa, más solidaria y, por otra, a luchar contra las desigualdades.

\section{El DIFíCIL RETO DE CONJUGAR LA COMPRENSIVIDAD Y LA DIVERSIDAD}

La universalización de la educación obligatoria en países desarrollados y de regímenes democráticos supuso una gran conquista que hoy exige algo más, una educación de calidad para todos que se sitúe lejos de las prácticas homogeneizadoras, de los criterios de eficacia y que no propugne fórmulas de diferenciación de los alumnos dentro de las escuelas (Gimeno, 1992). No se trata sólo de un cambio en la concepción de las instituciones educativas sino de un cambio nacido del propio desarrollo de los sistemas escolares democráticos que confluye con los cambios que el análisis de la realidad propia de la Educación Especial también promueve. Esta nueva concepción de "escuela para todos", nace por la manifestación de los deseos de los colectivos afectados por unas necesidades educativas diversas y por diversos intereses sociales que intentan dar una respuesta a las contradicciones y los problemas que la diversidad plantea. "Desde un enfoque dinámico y transformador de muchas contradicciones inherentes a la sociedad de consumo (clasificadora, homogeneizadora y estigmatizadora de las diferencias) se exige una respuesta que sólo puede dar una educación atenta y respetuosa con la diversidad, una escuela que, desde su proyección social, acepta la diversidad como uno de los grandes valores educativos" (Sánchez Palomino y Torres González, 2002: 70).

Como hemos venido poniendo de manifiesto, el hombre es un ser social por naturaleza, pero a la vez es un ser único. Esto nos lleva ineludiblemente a admitir la diversidad, pero también a reflexionar sobre el significado que ello tiene respecto a la sociabilidad, que es la otra cara para alcanzar los mínimos de persona humana. Ferrández (1997: 20) manifestaba al respecto que "individualidad y sociabilidad son dos acciones didácticas, y por lo tanto pedagógicas, que convergen en la formación 
de la personalidad". De hecho, las prácticas educativas suponen siempre marcos reguladores que reclaman respuestas coincidentes en los sujetos. Es decir, "las instituciones educativas obligan a las convergencias de las individualidades" (Gimeno, 2001: 235). El desarrollo madurativo de los individuos es un juego constante entre lo individual y lo social. Sin embargo parece ser que las cotas de socialización se alcanzan en función de una potenciación de las capacidades individuales, es decir, se llega a comportamientos sociales y socializadores gracias a que existe una posición crítica de carácter individual. La verdadera educación, por tanto, fortalecerá el pensamiento crítico personal, y desde él, se aprovecharán las aportaciones individuales que llegan al grupo, en cuyo seno se genera un conflicto sociocognitivo que obliga al juicio crítico y a toma de decisiones.

El referente, por tanto, en los procesos de enseñanza-aprendizaje será la individualidad de los sujetos que constituyen el grupo. Esa individualidad, "desde la autonomía personal y la consecuente responsabilidad asumida de manera crítica, garantiza el trabajo socializado en todas sus dimensiones" (Ferrández, 1997: 21). Sin embargo es preciso tener en cuenta, tal como plantea Gimeno (2001:235), que cuando la función socializadora es excesiva, provoca resistencia y tras un pretendido proyecto con fines de universalidad se ha tendido a promover la sumisión de lo que es diverso por naturaleza, intentando su normalización entendida como homogeneidad. En este sentido las instituciones educativas han promovido el etiquetaje y la clasificación de los sujetos en categorías. La educación tiene por tanto un difícil reto como es el de "sintetizar por un lado, los imperativos éticos de la individuación y, por otro, la necesidad de unir a los individuos en una red de interdependencias cognitivas, de proyectos y de sentimientos que creen comunidad" (Gimeno, 2001: 236).

Reivindicar una educación con talante igualitario (igualdad de oportunidades) y comprensivo no significa en ningún caso reclamar la uniformidad para todos sus alumnos, sino que "supone organizar la atención educativa en el respeto de las peculiaridades de cada estudiante y en el convencimiento de que las motivaciones, los intereses y la capacidad de aprendizaje son muy distintos entre los alumnos, debido a un complejo conjunto de factores, tanto individuales como de origen sociocultural que interactúan entre sí" (MEC 1989: 44-50).

La opción política de la Reforma Educativa del noventa suponía diseñar una enseñanza obligatoria que ofreciera las mismas oportunidades de instrucción y las mismas experiencias educativas a todos los alumnos con independencia de su posición social, posibilidades económicas, sexo, raza y otras características individuales. En este sentido, la escolaridad obligatoria se caracteriza por una educación que pretende ser comprensiva y a la vez diversificada, aspectos difíciles de conjugar y más aún de integrar, buscando el equilibrio entre ellos, pues una educación básica y común para todos los alumnos -no segregadora y que hace posible el principio de igualdad de oportunidades- debe articularse de forma coherente con aquel otro principio de satisfacer las necesidades educativas de todo el alumnado: atención a la diversidad. "El principio de enseñanza comprensiva debe, por tanto, complementarse con una oferta de enseñanza diversificada que proporcione satisfacción real a las necesidades educativas de los alumnos" (MEC, 1989: 119). 
"Esta opción política en favor de la comprensividad implica la necesidad, en el plano pedagógico, de articular procedimientos que integran la diversidad de alumnos que se van diferenciando progresivamente con el objeto de dar respuesta a sus intereses, capacidades y ritmos de aprendizaje" (Torres González, 1999: 136). Es por tanto necesario, que la organización de la enseñanza que adoptemos asegure un equilibrio real entre comprensividad y diversidad. Pero es aquí donde empiezan los problemas. Máxime cuando la diversidad del alumnado que puebla nuestras aulas es cada vez mayor. "En el momento actual, lo diverso ha llegado a constituirse en norma. La supuesta homogeneidad social, manejada no hace tanto tiempo como principio para su ordenación, no existe" (Casanova, 1999: 3). Es por ello, que desde nuestra perspectiva, el respeto a las diferencias y el enriquecimiento mutuo con las mismas debe constituir un planteamiento educativo básico. Con este propósito, y coincidiendo con Pérez Gómez (1995), debe sustituirse la lógica de la homogeneidad por la lógica de la diversidad.

Pero, ¿atender a la diversidad significa que mientras una parte del alumnado trabaja la literatura otra parte colorea mapas mudos? Esto se interrogaba un profesor en un debate sobre la cuestión. Contestar afirmativamente sin más consideraciones supondría un tratamiento claramente diferenciador de la diversidad. No obstante podría suceder que sólo una parte del alumnado trabajara la literatura y que eso ocurriera dentro de un marco escolar integrador. ¿De qué depende? ¿Cuál es el equilibrio entre la integración y la separación en grupos especiales para atender al alumnado con necesidades específicas o especiales para no contradecir la educación comprensiva? ¿Se ha sobrevalorado la integración física y temporal en el mismo espacio y en el mismo horario de todo el alumnado como punto de partida e inamovible para educar de manera integradora? En la práctica, se ha podido apreciar que en algunas ocasiones las integraciones físicas no evitan las marginaciones reales de determinados alumnos. ¿De qué depende?

En principio, conviene señalar que es bueno plantearse esta cuestión con ímpetu integrador, porque lo cierto es que en la mayoría de casos en los que diagnosticamos problemas de aprendizaje del alumnado estamos autodiagnosticando de hecho un problema organizativo que tenemos el profesorado: no sabemos qué hacer, o no podemos hacerlo, o no nos ponemos de acuerdo. Realmente no sabemos si tenemos "un niño con problemas" o "problemas con un niño". El sentido educativo de una actividad o estrategia de atención a la diversidad depende no tanto de sus componentes técnicos como del valor y significado que tienen en el contexto organizativo del centro: si la cultura organizativa del centro emite un mensaje integrador y promocionador o si emite un mensaje jerarquizador y clasificador.

Se puede comprobar cómo una actividad técnica y organizativamente similar adquiere un significado educativo diferente según el contexto del centro. Algunos indicadores del mismo pueden ser los siguientes:

- Valores y concepciones sobre la diversidad que dominan en la cultura de centro, en el proyecto de acción y en la gestión del centro. 
- Significado e intencionalidad que da el profesorado a una actividad de integración.

- Significado y valor que atribuye el alumnado participante, y el no participante, a la actividad.

- Grado de participación y acuerdo del alumnado implicado.

- Calidad de la integración física y temporal en el grupo ordinario.

Junto a este criterio básico se debe señalar otro factor del contexto del centro: los recursos disponibles. De este factor puede depender a veces que lo posible no sea lo mejor. Por tanto, "comprensividad y diversidad no son principios antagónicos sino complementarios. Si pretendemos una educación integradora y no discriminadora debemos asumir el reto de dar respuesta a la enorme complejidad de intereses, problemas y necesidades que se dan en la realidad educativa. Pero esta perspectiva no sería válida si no contribuye a compensar desigualdades haciendo efectivo el principio de igualdad de oportunidades que marca nuestra Constitución. No es una tarea fácil, ya que en la actualidad, tanto en la sociedad como en la escuela, existe una cultura selectiva que se arraiga en las propias convicciones sociales, culturales y pedagógicas de los profesores, de los alumnos y de los padres. En este sentido la cultura de la diversidad y del respeto a las diferencias no debe promoverse sólo en el ámbito educativo, sino que ha de formar parte e implicarse en los valores que se promueven en la sociedad. Hablaríamos de la diversidad como un valor social y político" (Torres González, 1999: 136). En este sentido, no debemos olvidar la brecha existente entre países desarrollados y poco desarrollados y que polariza a las poblaciones entre los que "todo pueden" y los que "la desesperanza" asoma como única perspectiva de vida. Es necesario, por tanto, intentar comprender que nos está sucediendo en la sociedad en su conjunto, en términos, por una parte, de ausencia de justicia como equidad, entendida como la igualdad de oportunidades en el desarrollo de las capacidades básicas, es decir, de habilidades efectivas para que las personas podamos trazarnos un proyecto de vida que no se reduzca a la subsistencia y, por otra, de replantear la tarea fundamental de la educación que no es otra que la de formar hombres y mujeres con capacidad crítica, autónomos y responsables para convivir conscientemente en la sociedad, capaces de evolucionar y adaptarse a la compleja realidad del mundo contemporáneo, a un mundo en constante y rápida mutación que implica la conformación de nuevas formas de enfrentar los procesos de enseñanza y de aprendizaje en la búsqueda de la calidad, de la excelencia.

Consideramos la importancia de aunar esfuerzos para tratar de progresar hacia la inclusión como medio de frenar los fortísimos procesos de exclusión social a los que, cada vez capas más amplias de nuestros conciudadanos y tristemente países enteros, están abocados (Echeíta, G. 2007); la postura de la UNESCO (2005) es clara cuando afirma que "la inclusión está relacionada con el acceso, la participación y los logros de todos los alumnos, con especial énfasis en aquellos que están en riesgo de ser excluidos o marginados". Esta inclusión la deberíamos enfocar, tal y como nos señala Puigdellivol no tanto en el alumno sino en el apoyo a la escuela, al profesorado y al alumno dentro del aula (2008: 27). 


\section{REFERENCIAS BIBLIOGRÁFICAS}

AINSCOW, M. (2001). Desarrollo de escuelas inclusivas: Ideas, propuestas y experiencias para mejorar las instituciones escolares. Madrid: Narcea.

BARTON, L. (1993). The struggle for Citizenship: The case of disabled people, disability. Handicap and Society, 8 (3), 235-248.

BERGER, P. L. y LUCKMANN, TH. (1984). La construcción social de la realidad. Buenos Aires: Amorrortu.

BERNSTEIN, B. (1977). Class, Codes and Control. Volumen 3: Towards a Theory of Educational Transmissions. Londres: Routledge \& Kegan Paul.

BERNSTEIN, B. (1991). Class, Codes and Control. Vol. 4. Towards a Theory of Educational Transmissions. Londres: Routledge \& Kegan Paul.

BORDIEU, P. Y PASSERON, J.C. (1977). La reproducción: Elementos para una teoría del sistema de enseñanza. Barcelona: Laia.

BRUNER, J. (1988). La importancia de la educación. Barcelona: Paidós.

BRUNER, J. (1997). La educación, puerta de la cultura. Madrid: Visor.

CAJIAO, F. (2001). La sociedad educadora. Revista Iberoamericana de Educación, 26, 17-33.

CANO, T. (2008). El maestro de audición y lenguaje en los centros T.I.C. En J.A. Torres y otros (Eds.). El maestro de audición y lenguaje en los centros T.I.C. Granada: Ada Book.

CARBONELL i PARÍS (2003). La integración de los diferentes. Boletín de la Asociación de Enseñantes con Gitanos, 21/22, 51-59.

CASANOVA, M. A. (1999). Educación para una sociedad plural. Organización y Gestión Educativa, 2, 3-7.

CASTELL, M. (1997). Flujos, redes e identidades: Una teoría crítica de la sociedad informacional. En M. Castells, R. Flecha, P. Freire, H. Giroux, D. Macedo y P. Willis (Eds.) Nuevas perspectivas críticas en educación (pp. 13-54). Barcelona: Paidós.

CASTELL, M. (1998). La era de la información. Volumen 2: El poder de la identidad. Madrid: Alianza.

DEL CARMEN, L. (2000). La atención a la diversidad: Una cuestión de valores. Aula de Innovación Educativa, 90, 7-11.

DELORS, J. (1996). La educación encierra un tesoro. Informe a la UNESCO de la Comisión Internacional sobre la educación para el siglo XXI. Madrid: Santillana, Ediciones UNESCO.

DURAND PONTE, V. (1989). Clases y sujetos sociales. Un enfoque crítico comparativo. México: UNAM.

DURKHEIM, E. (1975). Educación y Sociedad. Madrid: Lectura.

ECHEÍTA, G. (2007). Educación para la inclusión o educación sin exclusiones. Madrid: Narcea.

FERRÁNDEZ, A. (1997). La individualidad como fundamento de la igualdad de oportunidades: Bases para una propuesta curricular. En J.A. Torres González (Coord.). La innovación de la Educación Especial. Jaén: Servicio de Publicaciones de la Universidad de Jaén. 
FLECHA, R. (1997). Las nuevas desigualdades educativas. En M. Castells, R. Flecha, P. Freire, H. Giroux, D. Macedo y P. Willis (Eds.) Nuevas perspectivas críticas en educación (pp. 55-82). Barcelona: Paidós.

FLECHA, R. y TORTAJADA, I. (1999). Retos y salidas educativas en la entrada de siglo. En F. Imbernón y Otros (Coords.) La educación en el siglo XXI: Los retos del futuro inmediato (pp. 13-28). Barcelona: Biblioteca de Aula.

FORQUÍN, J. C. (1985). El enfoque sociológico del éxito y fracaso escolares: Desigualdad de éxito escolar y origen social. Educación y Sociedad, 3, 117-213.

GAIRÍN, J. y CASAS, M. (2003). La calidad en la educación. Barcelona: Cisspraxis.

GARCÍA, M. e ILLÁN, N. (1999). Las medidas ordinarias de atención a la diversidad: elemento clave para la escuela del siglo XXI. Organización y Gestión Educativa, 2, 30-34.

GIMENO, J. (1992). El currículum: ¿Los contenidos de la enseñanza o un análisis de la práctica? En J. Gimeno y A. I. Pérez, Comprender y transformar la enseñanza (pp. 137-170). Madrid: Morata.

GIMENO, J. (1999). La construcción del discurso acerca de la diversidad y sus prácticas. Aula de Innovación Educativa, 81-82, 67-72 y 73-78.

GIMENO, J. (2000). La construcción del discurso acerca de la diversidad y sus prácticas. En R. Alcudia y Otros. Atención a la diversidad. Barcelona: Graó.

GIMENO, J. (2001). Educar y convivir en la sociedad global. Madrid: Morata.

GIROUX, H. (1986). Teoría crítica e resistência em Educação. Petrópolis: Vózes.

LÓPEZ MELERO, M. (1997). La Educación Intercultural: El valor de la diferencia. En J. A. Torres González (Coord.). La innovación de la Educación Especial (pp. 173200). Jaén: Servicio de Publicaciones de la Universidad de Jaén.

LUNDGREN, U. P. (1992). Teoría del currículum y escolarización. Madrid: Morata. M.E.C. (1989). Libro Blanco para la Reforma del Sistema Educativo. Madrid: MEC.

MOUFFÉ, CH. (1994). La democracia radical. ¿Moderna o postmoderna? Revista Leviatán, 55, 81-94.

OLLERO, A. (1995). La dinámica de cambio en la España actual. Cuadernos de Pedagogía, 234, 74-79.

ORTEGA, J. A. (2000). Proyecto docente de Nuevas Tecnologías aplicadas a la educación. Inédito. Granada: Universidad de Granada.

PARRILLA, A. (2000). Proyecto docente e investigador. Inédito. Sevilla: Universidad de Sevilla.

PARSONS, T. (1982). El sistema social. Madrid: Alianza Universitaria.

PÉREZ GÓMEZ, A. I. (1991). Cultura escolar y aprendizaje relevante. Madrid: Educación y Sociedad.

PÉREZ GÓMEZ, A. I. (1992). Las funciones sociales de la escuela. De la reproducción a la reconstrucción crítica del conocimiento y la experiencia. En J. Gimeno y A. I. Pérez. Comprender y transformar la Enseñanza (pp. 17-33). Madrid: Morata.

PÉREZ GÓMEZ, A. I. (1995). La escuela, encrucijada de culturas. Investigación en la Escuela, 26, 7-24. 
PÉREZ TAPIAS, J. A. (1996). Claves humanísticas para una educación democrática. Madrid: Anaya.

PORRAS, R.; GARCÍA, M. y COTRINA, M. (2009). Posibilidades y límites del programa de ATAL en el marco de una escuela inclusiva. Revista de Educación Inclusiva, 2, (1), 11-28.

PUIGDELLIVOL, I. (2008). Comunidades de aprendizaje. Una escuela inclusiva (II). En Congreso Comunidades de Aprendizaje. Bilbao, 17-18 de febrero de 2008. Disponible en: http://www.jardunaldiak.net/pdf/COMUNIDADES DE APRENDIZAJE_ UNA_ESCUELA_INCLUSIVA.pdf (consultado el 20-01-2009).

SÁNCHEZ PALOMINO, A. y TORRES GONZÁLEZ, J. A. (2002). Las necesidades educativas especiales como alternativa a la categorización. En A. Sánchez Palomino y J. A. Torres González (Coords.), Educación Especial: Centros educativos y profesores ante la diversidad (pp. 63-81). Madrid: Pirámide.

SANTOS GUERRA, M. A. (2003). Trampas en educación. El discurso de la educación. Madrid: La Muralla.

STHENHOUSE, L. (1987). La investigación como base de la enseñanza. Madrid: Morata.

TEDESCO, J. C. (1995). El nuevo pacto educativo. Madrid: Anaya.

TORRES GONZÁLEZ, J. A. (Coord.) (1997). La innovación de la Educación Especial. Jaén: Servicio de Publicaciones de la Universidad de Jaén.

TORRES GONZÁLEZ, J. A. (1998) (Dir.). Intervención didáctica en Educación Especial. Jaén-Granada: ANDE/Universidad de Jaén.

TORRES GONZÁLEZ, J. A. (1999). Educación y Diversidad: Bases didácticas y organizativas. Málaga: Aljibe.

TORRES GONZÁLEZ, J. A. (2002). Educaçao e Diversidade. Brasil. Porto Alegre: ARTMED.

UNESCO (2005). Informe de seguimiento de la educación para todos. París: UNESCO.

WALKER, A. (1997). Introduction: The strategy of inequality. En A. Walker y C. Walker (Eds.). Britain divided. The growth of social exclusion in the 1980s and 1990 s. Londres: CPAG Ltd. 